\title{
A MONTE CARLO STUDY ON THE FIT OF A COST FUNCTION BY A FOURIER FLEXIBLE FUNCTIONAL FORM*
}

\author{
João Carlos F. Souza** \\ Geralclo S. Souza***
}

\section{Resumo}

Utilizamos o modelo de simulação de Chalfant e Gallant (1984) para estudar o ajuste de uma forma hexível de Fourier a uma função de custo. O objetivo é o de estimar uma elasticidade de substituição. Os dados que utilizamos apresentam erros nas variáveis exógenas e método de estimação é o de mínimos quadrados multivariados. Utilizamos no processo de estimação duas formas flexíveis de Fourier. Uma com 13 parámetros (FFF13) e outra com 22 (FFF22). Os vieses nas estimativas pontuais das elasticidades são pequenos para o ajuste com FFF13. As estatísticas $t$ clássicas não seguem, em geral, a distribuição de Student. Mesmo quando as estimativas são propriamente centradas e escalonadas existem casos onde a aproximação normal não se verifica.

\section{Abstract}

We use the simulation model of Chalfant and Callant (1985) to investigate the fit of a Fourier flexible form to a cost function when interest is to estimate clasticities of substitution. The data is subject to errors in variables and the estimation method is seemingly unrelated regressions. We use two approximating Fourier functional forms

\footnotetext{
* Research supported by CNPq

** Banco do Brasil, Cotec Presidéncia, Brasilia - DF

*** Enbrapa and Departarnento de Estatística, UnB, Brasília - DF
}

R. de Econometria Rio de Janeiro v. $15, \mathrm{n}^{\mathrm{0}} 2$, pp.31-54 Novembro/Março 1996 
A Monte Carlo study on the fit of a cost function

with 13 (FF'13) and 22 (FF'22) parameters. The biases in point estimation are negligible for F'F' 13 . The classical $t$ statistics do not follow, in general, the Student's distribution. Even when estimates are properly centered and scaled there are cases where the normal approximation does not hold.

Palavras-Chave: Flexible forms, factor demand systems, translog cost function, Fourier flexible form, estimation of elasticities

Código JEL: C15, D21

\section{Introduction.}

The classical use of a flexible functional form in econometrics to fit an indirect utility function in consumer theory, or a cost function in the theory of the firm, usually involves the specification of a model in a parametric family. For the theory of the firm one can achieve this purpose specifying the cost function as $C(p, y)=f\left(\alpha_{0}+\beta^{\prime} w+(1 / 2) w^{\prime} \Gamma w, v\right)$ where the function $f$ is known, $w$ is a vector of monotonically transformed prices $p$, and $v$ is a function of output $y$ that may depend on prices. The constant $\alpha_{0}$, the vector $\beta$, the matrix $\Gamma$, as well as any other unknown constants appearing in the function $v$, are parameters. A variant for consumer demand obtains dropping $v$ and letting $p$ be a vector of income normalized prices. Typical examples are provided by the generalized Box-Cox family (Berndt and Khaled (1979)) of which the class of Translog cost (indirect utility) functions is a limiting case. The objective of the econometric exercise of fitting an expenditure system is, usually, to estimate elasticities. One can completely specify these quantities with knowledge of the cost (indirect utility) function and its first and second order derivatives. From a statistical point of view the estimation of elasticities simplifies if the response model is taken from a parametric family and if the corresponding expenditure system is linear. This is the case of the theory of the firm with the use of the Translog. 
A parametric family of cost (indirect utility) functions is said to be flexible if it endows the estimation procedure with nonparametric properties, that is, if we have a reasonably close approximation to target population quantities (strong consistency, for example) even if the true response function is not a member of the family for any parameter choice. Relatively recent studies indicate that none of the classical parametric families can achieve flexibility in this sense. See White (1980), Guilkey, Lovell and Sickles (1983), Gallant (1981, 1982), and El Badawi, Gallant and Souza (1983). The reason for the lack of flexibility seems to be the failure of a Taylor series expansion to provide a uniform approximation simultaneously to a true response function and its first and second derivatives. In this context Gallant (1981) introduces the Fourier flexible form for indirect utility functions. The version for cost functions appeared in Gallant (1982). Essentially the Fourier flexible form superimposes a trigonometric polynomial to a Translog specification. The inclusion of sine and cosine terms endows the Translog family with the properties of Fourier series expansions. It is well known that a Fourier series can approximate a function in a Sobolev sense, that is, in a metric that allows a uniform approximation simultaneously for a function and its derivatives. If the observational model used to approximate the factor (consumer) demand system has additive and independent errors one can prove strong consistency of estimators of elasticities, conditionally on a realization of prices and other covariates, for any of the standard statistical procedures. For this result to hold it is necessary to introduce a dependence of the order of the approximating Fourier series on the sample size. The question of asymptotic normality in a context where the number of parameters grow with sample size is more delicate. Only recently this problem has been properly addressed and solved under very special circumstances. These circumstances hypothesize factor demand systems and multivariate least squares (seemingly unrelated regressions) with a known variance matrix. Sufficient conditions for asymptotic normality require the growth of the number of parameters at a rate slower than any 
power of the sample size. See Gallant and Souza (1991) and also Andrews (1994) for a flavor of the regularity conditions that may be involved in general and for a strategy to prove consistency and asymptotic normality in semiparametric models.

It has been argued in the econometric literature that actual factor demand systems do not show additive errors. The problem of errors in variables is intrinsic to such systems. Any realistic application of seemingly unrelated techniques will face this condition which have not been contemplated yet in theoretical studies. In this context Chalfant and Gallant (1985) use a reoriented central composite design and Monte Carlo simulation to investigate the performance of the Fourier flexible cost function. They restrict their attention to the study of statistical biases in the estimation of elasticities of substitution. Two variables define the factor space in their experiment: a measure of elasticity intensity $\sigma$ and a technology $\lambda$ chosen in the homothetic Box-Cox family (see also Box and Cox, 1964). They fit a quadratic form in $\sigma$ and $\lambda$ to the response surface of biases generated by elasticities of substitution estimated via seemingly unrelated regressions. Here we extend Chalfant and Gallant's study. We investigate the distributions of the estimates and deepen the discussion on statistical biases. Our objective, basing conclusions on Monte Carlo evidence, is to provide specific recommendations and warnings in regard to the practical use of the Fourier flexible cost function. We organize our presentation as follows. In Section 2 we introduce the family of cost functions we use later. These are the general BoxCox form and the Fourier fexible form. In Section 3 we describe in detail the experiment of Chalfant and Gallant (1985). In Section 4 we present our simulation results. We base our analysis on the absolute biases observed in estimates of substitution elasticities and in two measures of goodness of fit: the chi-square test statistic and the Shapiro-Wilk test statistic. Finally we summarize our findings in Section 5. 


\section{Cost Functions.}

Consider a production scheme involving $n$ factor inputs. Let $p^{\prime}=\left(p_{1}, \cdots, p_{n}\right)$ be a vector of input prices and let $q^{\prime}=\left(q_{1}, \cdots, q_{n}\right)$ be the vector of input quantities. The total expenditure resulting from the choice of quantity vector $q$ is $p^{\prime} q$. Let $y=f\left(q_{1}, \cdots, q_{n}\right)$ be the production function. The producer's problem, given a vector of factor input prices $p$ and a level of output $y$, is to minimize total expenditure $p^{\prime} q$ subject to $f\left(q_{1}, \cdots, q_{n}\right)=y$. The minimum expenditure $C(p, y)$ corresponding to the solution $q(p, y)$ is the producer's cost function. The vector $q(p, y)$ defines the set of factor demand functions.

The theory of the firm may impose regularity conditions on $C(p, y)$. A minimal set of regularity conditions will impose linear homogeneity in prices, monotonicity in prices and output, and concavity in prices. A cost function $C(p, y)$ is said to be linear homogeneous if $C(\tau p, y)=\tau C(p, y)$ for any $\tau>0$, monotonic if $\nabla_{p, y} C(p, y)>0$, and concave if $\nabla_{p}^{2} C(p, y)$ is negative semidefinite. Concavity and linear homogeneity imply that $\nabla_{p}^{2} C(p, y)$ has rank at most $n-1$ with the price vector $p$ being an eigenvector of root zero. A simplifying assumption on functional form imposes homotheticity (a general form of constant returns to scale), that is $C(p, y)=h(p) \phi(p)$ where $h(p)$ is linear homogeneous.

A linear homogeneous technology (cost function) will satisfy Shephard's lemma, that is $q(p, y)=\nabla_{p} C(p, y)$ or, equivalently, $S_{i}=\partial C(p, y) / \partial p_{i} / C(p, y)$, where $S_{i}$ is the cost share of the $i$ th input factor. Typically the econometricians that deal with expenditure systems will specify a parametric form for $C(p, y)$ and postulate an observational multivariate (possibly nonlinear) statistical model $s_{i}=\partial C(p, y) / \partial p_{i} / C(p, y)+\varepsilon_{i}, i=1 \cdots n-1$. In this latter expression $s_{i}$ represents an observed share, $p$ are observed prices, and one share equation has been removed to avoid a singular model.

In some instances is more convenient to express the cost function, prices, and output in $\log$ (base e) form. If $g(l, v)$ represents the 
$\log$ cost function, $l=\ln p$, and $v=\ln y$, the observational model becomes $s_{i}=\partial g(l, v) / \partial l_{i}+\varepsilon_{i}$.

The most common statistical technique used in an attempt to determine $C(p, y)$ or $g(l, v)$ statistically is seemingly unrelated regressions. This estimation technique proceeds as follows. For observation $t=1 \cdots T$ let $s_{\iota}^{\prime}=\left(s_{1 l}, \cdots, s_{(n-1) \iota}\right)$ be the vector of shares and $x_{t}^{\prime}=\left(l_{\imath}^{\prime}, v_{\iota}\right)$. We can write $s_{t}=\phi\left(x_{\imath}, \theta\right)+\varepsilon_{\imath}$ where $\varepsilon_{\iota}$ is an $n-1$ dimensional error vector and $\theta$ is a parameter derived from a completely specified cost function. Let $S(\theta, V)=$ $\sum_{\ell=1}^{T}\left(s_{\iota}-\phi\left(x_{\iota}, \theta\right)\right)^{\prime} V^{-1}\left(s_{\iota}-\phi\left(x_{\iota}, \theta\right)\right)$ and let $\Psi(\theta)=0$ be a set of restrictions on $\theta$. The seemingly unrelated regressions estimate of $\theta$ obtains first finding $\bar{\theta}$ that minimizes $S(\theta, I)$ subject to $\Psi(\theta)=0$, putting $\hat{V}=(1 / T) \sum_{l=1}^{T}\left(s_{\iota}-\phi\left(x_{t}, \bar{\theta}\right)\right)^{\prime}\left(s_{\iota}-\phi\left(x_{t}, \bar{\theta}\right)\right)$ and then minimizing $S(\theta, \hat{V})$ subject to $\Psi(\theta)=0$. Iteration of this process, upon convergence, yields maximum likelihood estimates under normal errors. See Gallant (1987).

As Gallant (1982) points out the main objective of the econometric attempt to fit share equations focus on the assessment of elasticities of substitution

$$
\sigma_{i, j}=\left(C(p, y) \frac{\partial^{2} C(p, y)}{\partial p_{i} \partial p_{j}}\right) /\left(\frac{\partial C(p, y)}{\partial p_{i}} \frac{\partial C(p, y)}{\partial p_{j}}\right), i, j=1, \cdots, n
$$

and price elasticities $\eta_{i j}=S_{j} \sigma_{i j}$. Let $\Sigma=\left(\sigma_{i j}\right)$. The assumption of concavity in prices for $C(p, y)$ is equivalent to $\Sigma$ being negative semidefinite. Linear homogeneity implies that the share vector $S$ must be an eigenvector of root zero for $\Sigma$.

\subsection{The Box-Cox Cost Function.}

Berndt and Khaled (1979) present in detail the generalized BoxCox parametric family of cost functions. We concern ourselves here 
only with homothetic technologies. Imposing this condition and linear homogeneity in prices the generalized Box-Cox family reduces to

$$
C_{\lambda}(p, y)=\left[(2 / \lambda) \sum_{i} \sum_{j} \gamma_{i j} p_{i}^{\lambda / 2} p_{j}^{\lambda / 2}\right]^{1 / \lambda} \phi(y) .
$$

The matrix $\Gamma=\left(\gamma_{i j}\right)$ is symmetric and $\lambda>0$. For these cost functions the elasticities of substitution do not depend on output. Since our primary interest is only in the estimation of elasticities of substitution we assume output to be unit and drop the term $\phi(y)$.

Familiar technologies in the Box-Cox family are the Square Root Quadratic $(\lambda=2)$ and the Generalized Leontief $(\lambda=1)$. The Translog obtains taking the limit as $\lambda \rightarrow 0$. The Translog cost function (in $\log$ form) is

$$
g(l, v)=\alpha_{0}+\alpha^{\prime} l+\frac{1}{2} l^{\prime} \Gamma l+\left(\beta+\sum_{i=1}^{n} l_{i}\right) v+\frac{\theta}{2} v^{2}
$$

where we must impose $\Sigma_{i} \alpha_{i}=0$ and $\Sigma_{j} \gamma_{i j}=0$ for linear homogeneity in prices. The terms in $v$ disappear when output is unity.

If $\lambda>0$ the expressions for shares and elasticities of substitution are

$$
\begin{aligned}
& S_{i}=\left(2 p_{i}^{\lambda / 2} \sum_{j=1}^{n} \gamma_{i j} p_{j}^{\lambda / 2}\right) /\left(\lambda C^{\lambda}(p, y)\right), \quad i=1, \cdots, n \\
& \sigma_{i i}=1-\lambda+\gamma_{i i} \frac{p_{i}^{\lambda}}{S_{i}^{2}} C_{\lambda}^{-\lambda}(p, y)+\frac{\lambda}{2 S_{i}}-\frac{1}{S_{i}}, \quad i=1, \cdots, n \\
& \sigma_{i, j}=1-\lambda+\gamma_{i j} \frac{p_{i}^{\lambda / 2} p_{j}^{\lambda / 2}}{S_{i} S_{j}} C_{\lambda}^{-\lambda}(p, y), \quad i \neq j
\end{aligned}
$$


and for $\lambda=0$

$$
\begin{aligned}
S_{i} & =\alpha_{i}+\sum_{j=1}^{n} \gamma_{i j} l_{j}, \quad i=1, \cdots, n \\
\sigma_{i i} & =1+\frac{\gamma_{i i}}{S_{i}^{2}}-\frac{1}{S_{i}}, \quad i=1, \cdots, n \\
\sigma_{i j} & =1+\frac{\gamma_{i j}}{S_{i} S_{j}}, \quad i \neq j .
\end{aligned}
$$

Using the normalization rule $C_{\lambda}(p)=1$, given values for $\lambda, \sigma_{i j}$, $p_{i}$, and $S_{i}$, one can solve Equations (1)-(6) to find a unique set of $\gamma_{i, j}$. Later we use this fact to derive population technologies. In this exercise the specification of the substitution matrix $\Sigma$ will imply values for $S_{i}$. Notice that with knowledge of $\gamma_{i j}$ we can use Equations (1) and (4) to generate share data.

\subsection{The Fourier Flexible Form.}

Gallant (1982) introduces the Fourier flexible form for the analysis of factor demand systems as follows. As before let $x^{\prime}=\left(l^{\prime}, v\right)$ where now $l_{i}=\ln p_{i}+\ln a_{i}$ and $v=\ln y+\ln a_{n+1}$. The constants $a_{1}, \cdots, a_{n+1}$ are location quantities chosen such that $x$ becomes a vector with all coordinates strictly positive. For the prices components one may take $\ln a_{i}=-\min _{i} \ln p_{i}+\zeta$ for any choice of $\zeta>0$. The functional form of the Fourier flexible form with $I$ parameters is

$$
\begin{aligned}
g_{K}(x, \theta) & =u_{0}+b^{\prime} x+\frac{1}{2} x^{\prime} B x+\sum_{\alpha=1}^{A}\left\{u_{0 \alpha^{\cdot}}+\sum_{j=1}^{J}\left[u_{j \alpha} \cos \left(j \tau k_{\alpha}^{\prime} x\right)-\right.\right. \\
& \left.\left.-v_{j \alpha} \sin \left(j \tau k_{\alpha}^{\prime} x\right)\right]\right\}
\end{aligned}
$$


with

$$
B=-\tau^{2} \sum_{\alpha=1}^{n} u_{0 \alpha} k_{\alpha} k_{\alpha}^{\prime}
$$

Here $u_{0}, u_{0 \alpha}, b, B, u_{j \alpha}$, and $v_{j \alpha}$ are parameters, $k_{\alpha}$ is a vector with integer components - a multi-index, and $\tau$ is a scale parameter chosen to make each $x$ a point of $(0,2 \pi)^{n+1}$. The vector $\theta$ represents the collection of parameters written down in some order. In this paper we use $\zeta=10^{-5}$ and $\tau=6 / \max _{i} x_{i}$. Since output is taken to be unit, in our instance the vector $x$ is equal to $l$.

A deep philosophical difference between the Fourier flexible form and other parametric cost functions lies in the dependence of $I$ on the sample size $T$. We emphasize this dependence here writing $I^{\prime}=I_{T}$. The Fourier flexible form $\boldsymbol{g}_{K}(x, \theta)$ approximates a true cost function in the Sobolev sense if $\Gamma_{T} \rightarrow \infty$ as $T \rightarrow \infty$. This result poses some problems in applications. It is not clear in the literature of the subject how one should optimally choose $I_{T}$ and therefore $A, J$, and the multi-indices $k_{\alpha}$.

The Fourier flexible form will approximate a linear homogeneous cost function if we impose $\Sigma_{i} b_{i}=1$ and keep only terms corresponding to multi-indices $k_{\alpha}$ for which $\Sigma_{i} k_{\alpha i}=0$.

The Fourier flexible form expenditure system is linear since shares can be expressed as $S_{i}=\partial g_{K_{T}}(x, \theta) / \partial x_{i}=g_{i}^{\prime} \theta$. Since also $\partial^{2} g_{K T}(x, \theta) / \partial x_{i} \partial x_{j}=h_{i j}^{\prime} \theta$ we can compute elasticities of substitution as

$$
\sigma_{i i}(\theta)=1+\frac{h_{i i}^{\prime} \theta}{\left(g_{i}^{\prime} \theta\right)^{2}}-\frac{1}{g_{i}^{\prime} \theta} \quad \text { and } \quad \sigma_{i j}(\theta)=1+\frac{h_{i j}^{\prime} \theta}{\left(g_{i}^{\prime} \theta\right)\left(g_{j}^{\prime} \theta\right)} \quad i \neq j .
$$

It follows that if an estimator $\hat{\theta}$ is available, via seemingly unrelated regressions for example, we can estimate consistently the true elasticity of substitution $\sigma_{i j}$ by $\sigma_{i j}(\hat{\theta})$ and try to approximate its variance by $\nabla^{\prime} \sigma_{i j}(\hat{\theta}) \hat{\Omega} \nabla \sigma_{i j}(\hat{\theta})$ where $\hat{\Omega}$ is an estimate of the variance matrix of $\hat{\theta}$. One can compute price elasticities estimators in 
an analogous manner. See Gallant (1982) and El Badawi, Gallant and Souza (1983) for more details on this approach.

In our simulation study we use, as in Chalfant and Gallant (1985), two Fourier flexible forms: one for which $K_{T}=13(F F F 13)$ and another for which $K_{T}=22(F F F 22)$. In both cases the true technology is a function of a price vector of dimension three. For FFF22 we take $A=6$ and $J=1$. The choices of multi-indices ar $k_{1}^{\prime}=\left(\begin{array}{lll}0 & 1 & -1\end{array}\right), k_{2}^{\prime}=\left(\begin{array}{ll}1-1 & 0\end{array}\right), k_{3}^{\prime}=\left(\begin{array}{lll}1 & 0 & -1\end{array}\right), k_{4}^{\prime}=\left(\begin{array}{lll}1 & -2 & 1\end{array}\right)$, $k_{5}^{\prime}=\left(\begin{array}{lll}1 & 1 & -2\end{array}\right)$, and $k_{6}^{\prime}=(2-1-1)$. The first three have norm two and the last three norm four. The norm of a multi-index is the sum of the absolute values of its components. The resulting (FFF22) form is

$$
\begin{aligned}
& g_{22}(x, \theta)=u_{0}+b^{\prime} x+\frac{1}{2} x^{\prime} B x \\
& \quad+u_{01}+2\left\{u_{1} \cos \left[\tau\left(x_{2}-x_{3}\right)\right]-v_{1} \sin \left[\tau\left(x_{2}-x_{3}\right)\right]\right\} \\
& \quad+u_{02}+2\left\{u_{2} \cos \left[\tau\left(x_{1}-x_{2}\right)\right]-v_{2} \sin \left[\tau\left(x_{1}-x_{2}\right)\right]\right\} \\
& \quad+u_{03}+2\left\{u_{3} \cos \left[\tau\left(x_{1}-x_{3}\right)\right]-v_{3} \sin \left[\tau\left(x_{1}-x_{3}\right)\right]\right\} \\
& \quad+u_{04}+2\left\{u_{4} \cos \left[\tau\left(x_{1}-2 x_{2}+x_{3}\right)\right]-v_{4} \sin \left[\tau\left(x_{1}-2 x_{2}+x_{3}\right)\right]\right\} \\
& \quad+u_{05}+2\left\{u_{5} \cos \left[\tau\left(x_{1}+x_{2}-2 x_{3}\right)\right]-v_{5} \sin \left[\tau\left(x_{1}+x_{2}-2 x_{3}\right)\right]\right\} \\
& \quad+u_{06}+2\left\{u_{6} \cos \left[\tau\left(2 x_{1}-x_{2}-x_{3}\right)\right]-v_{6} \sin \left[\tau\left(2 x_{1}-x_{2}-x_{3}\right)\right]\right\}
\end{aligned}
$$

The parametrization above for FFF22 leads to a singular design matrix. To avoid this we reparametrize the elements of $B$ and absorb the terms $u_{0 a}$ in the constant term. On $B$ we impose symmetry and homogeneity.

The version FFF13 obtain from FFF22 reducing the order of the trigonometric polynomial. Here we consider only multi-indices with norm two. This is equivalent to set the coefficients $u_{04}, u_{05}, u_{06}, u_{4}$, 
$v_{4}, u_{5}, v_{5}, u_{6}$, and $v_{6}$ to zero in the expression for FFF22. Also

$$
B=-\tau^{2}\left(\begin{array}{ccc}
-u_{02}-u_{03} & u_{02} & u_{03} \\
u_{02} & -u_{01}-u_{02} & u_{01} \\
u_{03} & u_{01} & -u_{01}-u_{03}
\end{array}\right)
$$

and the parametrization is identifiable.

\section{Experimental Design.}

Basing their claims on the empirical evidence of past work Chalfant and Gallant (1985) argue that the presence of bias, and its relative size, in the statistical estimation of elasticities, may depend on the nature of the true technology, on the particular pattern of the elasticity matrix, and on the magnitude of each elasticity being estimated. In this context they design a response surface experiment depending on two quantitative factors: Technology - measured by variate $\lambda$, and Elasticity - measured by variate $\sigma$. The design space is the rectangle $[0,2] \times[0,2]$. Each choice of $(\sigma, \lambda)$ defines a technology $C_{\lambda}\left(p_{1}, p_{2}, p_{3}\right)$ in the homothetic Box-Cox family for which the matrix of elasticities of substitution is $\Sigma(\sigma)$. They use a total of nine design points chosen as explained below. The idea is to fit the Fourier flexible form to data generated according to each of these "true" technologies and investigate the nature of the response surface defined by the absolute biases resulting from the estimation process. Of particular concern is the presence of a ridge of increasing bias in any particular direction $(\sigma \lambda)$. They compute $\Sigma(\sigma)$ as follows. The own price elasticities are taken to be equal to $-\eta$ for all three prices. The diagonal elements of $\Sigma(\sigma)$ are then $\sigma_{i i}=-\eta / S_{i}$. The off diagonal elements have the same magnitude $\sigma$ and $\Sigma$ has one of the three patterns

$$
I:\left(\begin{array}{ccc}
\sigma_{11} & \sigma & \sigma \\
\sigma & \sigma_{22} & -\sigma \\
\sigma & -\sigma & \sigma_{33}
\end{array}\right) \quad I I:\left(\begin{array}{ccc}
\sigma_{11} & \sigma & -\sigma \\
\sigma & \sigma_{22} & \sigma \\
-\sigma & \sigma & \sigma_{33}
\end{array}\right)
$$


A Monte Carlo study on the fit of a cost function

$$
I I I:\left(\begin{array}{ccc}
\sigma_{11} & -\sigma & \sigma \\
-\sigma & \sigma_{22} & \sigma \\
\sigma & \sigma & \sigma_{33}
\end{array}\right)
$$

The actual pattern used for each combination $(\sigma \lambda)$ was chosen randomly.

Given a value of $\sigma \neq 0, \Sigma$ is completely determined by the placement of the negative sign. We illustrate this construction when

$$
\Sigma=\left(\begin{array}{ccc}
-\frac{\eta}{S_{1}} & \sigma & \sigma \\
\sigma & -\frac{\eta}{S_{2}} & -\sigma \\
\sigma & -\sigma & -\frac{\eta}{S_{3}}
\end{array}\right)
$$

It follows (from $\Sigma S_{i}=1$ and $\Sigma S=0$ that

$$
\left\{\begin{array}{l}
-\eta+0 S_{1}+\sigma S_{2}+\sigma S_{3}=0 \\
-\eta+\sigma S_{1}+\sigma S_{2}+-\sigma S_{3}=0 \\
-\eta+\sigma S_{1}+-\sigma S_{2}+-0 S_{3}=0 \\
-0 \eta+S_{1}+S_{2}+S_{3}=1
\end{array}\right.
$$

The solution for this system is $S_{1}=3 / 5, S_{2}=S_{3}=1 / 5$ and $\eta=$ $2 \sigma / 5$. Thus for Pattern I

$$
\Sigma(\sigma)=\frac{\sigma}{3}\left(\begin{array}{ccc}
-2 & 3 & 3 \\
3 & -6 & -3 \\
3 & -3 & -6
\end{array}\right)
$$

For Patterns II and III we obtain, respectively,

$$
\Sigma(\sigma)=\frac{\sigma}{3}\left(\begin{array}{ccc}
-6 & 3 & -3 \\
3 & -2 & 3 \\
-3 & 3 & -6
\end{array}\right) \text { and } \Sigma(\sigma)=\frac{\sigma}{3}\left(\begin{array}{ccc}
-6 & -3 & 3 \\
-3 & -6 & 3 \\
3 & 3 & -2
\end{array}\right) \text {. }
$$


Notice that these three matrices are negative definite with rank two. At this point it is important to remark that when $\sigma=0$ a random choice among Patterns I, II, and III was used to decide which share should have value $3 / 5$.

The design points in the factor space where chosen as follows. Consider first the central composite rotatable design in Cochran and Cox (1957). This is a design commonly used in experimental statistics to approximate a response function by a quadratic form. The nine vectors $(0-\sqrt{2}),(00),(11),(0 \sqrt{2}),(-11)$, $(-1-1),(1-1),(\sqrt{2} 0)$, and $(-\sqrt{2} 0)$ define the design. The transformation $\left(\frac{x+\sqrt{2}}{\sqrt{2}}, \frac{y+\sqrt{2}}{\sqrt{2}}\right)$ maps the central composite into the square $[0,2] \times[0,2]$ producing the new design points $(\sigma, \lambda):(10)$, (11), (1.70711 1.70711), (12), (0.292891.70711), (0.292890.29289), (1.707110.29289), (21), and (01). This is the layout we will use. We refer to these points as design points $1-9$ respectively. Known technologies in the layout are the Translog - Design 1, Leontief - Design 9, Square Root Quadratic - Design 4, and Generalized Leontief - Design 2 and 8.

It is clear from Equations (1)-(6) of Section 2 and from the normalization rule $C_{\lambda}(p)=1$ at $p=p_{0}$ that a design point $(\sigma, \lambda)$ uniquely determines $C_{\lambda}(p)$ with the choice $\Sigma=\Sigma(\sigma)$.

Chalfant and Gallant (1985) consider two price series to generate data in the Monte Carlo process. The idea is to mimic the behavior of expected and actual prices so that share data is simulated with expected prices and fit to actual prices. A typical case of errors in variables and non additive regression errors. The series of actual prices $p_{t}$ is fixed and is generated according to the model $\ln p_{t}=\beta+u_{t}$, $u_{t}=R u_{t-1}+\varepsilon_{t}$, where $\beta^{\prime}=(0.09760778-0.007215130 .37562201)$,

$$
R=\left(\begin{array}{rrr}
0.56627 & 0.055096 & -0.017574 \\
0.13356 & 0.502859 & 0.129266 \\
-0.14791 & 0.110825 & 0.934661
\end{array}\right)
$$

and $\varepsilon_{t}$ is a three dimensional multivariate normal random vector 
A Monte Carlo study on the fit of a cost function

with mean zero and variance $V=F^{\prime} F$. Here

$$
F=\left(\begin{array}{ccc}
-0.00135824 & -0.08386093 & -0.00054760 \\
0.00067686 & 0.00017299 & -0.02817099 \\
-0.01265727 & 0.00206979 & -0.00030284
\end{array}\right)
$$

For $p_{0}$ we take $\exp (\beta)$. This is the price vector used to obtain the population technologies in Table 1 . The (vector) stochastic process above was adapted by Chalfant and Gallant (1985) from a real price series studied in Berndt and Wood (1975).

Following Huber (1981) $K_{T} \approx T^{2 / 3}$. In this context a basic sample of 25 observed prices is generated to fit FFF13 and extended to 48 to fit FFF22. With only two shares retained the number of effective observations in each case becomes 50 and 96 respectively.

The series of expected prices $\hat{p}_{t}$ is a function of observed prices $p_{t}$. We take $\ln \left(\hat{p}_{t}\right)=\ln \left(p_{t}\right)+\zeta_{t}$ where $\zeta_{t}=\beta * y_{t}$. Here $y_{t}$ is a multivariate normal random vector with mean zero and variance matrix $\alpha I$. Shares are generated using the formula $s_{i t}=\partial C_{\lambda}\left(\hat{p}_{t}\right) / \partial p_{i} / C_{\lambda}\left(\hat{p}_{t}\right)$ which is Shephard's lemma. Chalfant and Gallant (1985) genrate 5000 replications and set $\alpha$ to 0.1 . This choice of $\alpha$, in their case, allows estimation of elasticities with accuracy of three digits when the true technology is the Translog. Here we take 1000 Monte Carlo replications. We achieve the same accuracy for the Translog case with $\alpha=0.01$ for both fits (FFF13 and FFF22). The number of replications we choose is in accordance with the bootstrap literature, see Efron (1990) and Efron and Tibshirani (1993), and keeps the simulation process at a $\mathrm{PC}$ manageable size.

\section{Simulation Results.}

Our simulation study mimics the basic structure (model) of Chalfant and Gallant (1985). We use SAS - ETS to compute seemingly unrelated regressions, SAS - PROC IML to compute standard errors of elasticities of substitution and nonparametric density estimates, and SAS - STAT to evaluate empirical distributions. In 
Subsection 4.1 we present our findings in regard to biases in estimation. In Subsection 4.2 we show our results regarding the adequacy of large sample distribution approximations.

\subsection{Biases in Estimation.}

The variable of concern here is the absolute bias $\left|\sigma_{i j}-\bar{\sigma}_{i j}\right|$ where $\sigma_{i j}$ is the true elasticity of substitution and $\bar{\sigma}_{i j}$ is an average of 1000 seemingly unrelated estimates $\hat{\sigma}_{i j}$.

Table 2 shows the evolution of two summary statistics computed from the basic results reported in Félix Souza (1993). Those are the average absolute bias per technology $(\lambda)$ and the average absolute bias relative to the Translog which functions as a base case or control. The overall impression is that FFF13 has a better performance than FFF22. The worst case for FFF13 is technology five which on average shows a bias close to 0.03 . This is about eight times the bias we measure when we use FFF13 and the data is generated according to the Translog. The fit for FFF22 produces four bad estimates of elasticities. Two of those seem to be definite outliers: technologies four and eight. The average absolute biases are 0.08 and 0.14 respectively. These figures are approximately 45 and 77 times the average bias we measure when we fit FFF22 to data generated from the Translog. Chalfant (1983) also reports an increase in bias when using the FFF22 but our results are more dramatic. He attributes the misfit to errors in variables. In our case other sources of instability may also be confounded in the process. Potential candidates are specification bias and multicollinearity. We dit not notice any significant effect due to the difference in patterns present in $\Sigma$.

The response surface analysis for the absolute bias is shown in Tables 3 and 4 . Model FFF13 passes the lack of fit test and the stationary point is a saddle point outside the region under study. This is an indication of good performance. Model FFF22 does not pass the lack of fit test but a ridge of increase in response is observed in the direction of $(\sigma=1.956 \lambda=1.292)$ which is close to Design 8 . 
A Monte Carlo study on the fit of a cost function

These results are in close agreement with the findings of Table 2.

\subsection{Probability Distributions.}

To identify directions of poor large sample approximations (via response surface analysis) in the technology factor space we compute two measures of goodness of fit. A measure we call $X^{2}$, which is the chi-square test statistic, and the Shapiro-Wilk test statistic $W$. For model FFF13 the variable $X^{2}$ measures the overall discrepancy from the fit of a $t$ distribution with $39 \mathrm{df}$ to observed ratios of the form $t_{i j}=\left(\hat{\sigma}_{i j}-\sigma_{i j}\right) / s\left(\hat{\sigma}_{i j}\right)$ where $s\left(\hat{\sigma}_{i j}\right)$ is the seemingly unrelated regressions estimate of the standard error of $\hat{\sigma}_{i j}$. For FFF22 variable $X^{2}$ measures a similar quantity for the $t$ distribution with $79 \mathrm{df}$. We consider in both cases 14 non overlapping classes. The boundaries of these classes are set in such way to produce the same probabilities under $t_{39}$ and $t_{79}$ respectively. The Shapiro-Wilk test statistic $W$ measures departure from normality when we consider the variate $\hat{\sigma}_{i j}$.

The empirical probabilities associated with $t_{i j}$ in many cases differ markedly from tables of the $t$ distribution. On the other hand the behavior of $\hat{\sigma}_{i j}$ is close to normality. Figures 1 and 2 illustrate these facts showing density estimates for some elasticities in Design 8. With a few exceptions the pattern is typical of other designs. The visual impression is confirmed by $X^{2}$ and $W$. Overall the better approximations are for FFF13.

We show the response surface analysis for $\ln X^{2}$ and $W$ in Tables 5, 6, 7 and 8. In the case of $\ln X^{2}$ FFF13 passes the lack of fit test and the stationary point with coordinates $(\sigma=1.288618 \lambda=0.9456335)$ is a maximum. This is close to the Generalized Leontief (Design 2). The model FFF22 does not pass the lack of fit test. The variable $W$ shows opposite results. FFF 13 does not pass the lack of fit and FFF22 does. In this instance the stationary point is a minimum and has coordinates $(\sigma=0.9812826 \lambda=0.871032)$. As with $\ln X^{2}$ we see that the direction of worst results is close to the Generalized 
Leontief. For both response variables, whenever the lack of fit test fails, we notice a ridge in the direction of this same technology.

As a final comment in regard to the statistics $t_{i j}$ we point out our impression that the bias in the estimation of both, $\sigma_{i j}$ and $s\left(\hat{\sigma}_{i j}\right)$, rather than the polynomial rate of dependence to the sample size, may the cause for the poor $t$ distribution approximations.

\section{Conclusion.}

The Fourier flexible form seems to perform very well for $I_{T}=$ 13, particularly in regard to point estimation of elasticities. The inclusion of additional trigonometric terms seems to lead to unstable estimates. The classical Wald test statistics based on seemingly unrelated estimates do not follow a $t$-distribution. The better distribution results obtained with the Shapiro-Wilk test statistic, which properly centers and scales the estimates $\hat{\sigma}_{i j}$, is an indication that the use of bootstrap techniques to correct for bias and to set confidence intervals will provide a morc reliable statistical inference than the classical delta method. In general our analysis indicate that distribution results are particularly bad in the direction of Generalized Leontief cost functions. The discrepancies observed are too large to be attributed to errors in variable alone.

Submetido em Abril de 1995. Revisado em Agosto de 1995.

\section{Referências}

Andrews, D.W.K. 1994. "Asymptotics for semiparametric econometric models via stochastic equicontinuity". Econometrica, 62: 43-72.

Berndt, E.R. \& M.S. Khaled 1979. "Parametric productivity measurement and choice among flexible functional forms". Journal of Political Economy, 87: 1220-1245. 
A Monte Carlo study on the fit of a cost function

Berndt, E.R. \& D.O. Wood 1975. "Technology, prices, and the derived demand for energy". Review of Economics and Statistics, 57: 259-268.

Box, G.E.P. \& D.R. Cox 1964. "An analysis of transformations". Journal of the Royal Statistical Society, B-26: 651-676.

Chalfant, J.A. 1983. "Choosing Among Flexible Functional Forms: An Application of the Generalized Box-Cox and the Fourier Flexible Forms to U.S. Agriculture". PhD dissertation, Department of Economics and Business and Department of Statistics, North Carolina State University, Raleigh, North Carolina, USA.

Chalfant, J.A. \& A.R. Gallant 1985. "Estimating substitution elasticities with the Fourier cost function". Journal of Econometrics, 28: 205-222.

Cochran, W.G. \& G.M. Cox 1957. Experimental Designs. Wiley, New York.

Efron, B. 1990. "More efficient bootstrap computations". Journal of the American Statistical Association, 37: 79-89.

Efron, B. \& R.J. Tibshirani 1993. An Introduction to the Bootstrap, Chapman and Hall, New York.

El Badawi, L.; A.R. Gallant \& G. Souza 1983. "An elasticity can be estimated consistently without a priori knowledge of functional form". Econometrics, 51: 1731-1751.

Félix Souza, J.C. 1993. "Uma Análise Empírica do Comportamento de Elasticidades de Substituição obtidos via a Forma Flexível de Fourier", M.Sc. dissertation, Department of Statistics, University of Brasília - DF, Brazil.

Gallant, A.R. 1981. "On the bias in flexible forms and an essentially unbiased form: the Fourier flexible form". Journal of Econometrics, 15: 211-245.

Gallant, A.R. 1982. "Unbiased determination of production technologies". Journal of Econometrics, 20: 285-323. 
Gallant, A.R. 1987. Nonlinear Statistical Models, John Wiley, New York.

Gallant, A.R. \& G. Souza 1991. "On the asymptotic normality of Fourier flexible form estimates". Journal of Econometrics, 50: 329-353.

Guilkey, D.K.; C.A.K. Lovell \& R. Sickles 1983. "A comparison of the performance of three flexible functional forms". International Economic Review, 21: 137-147.

Huber, P.J. 1981. Robust Statistics, Wiley, New York.

White, H. 1980. "Using least squares to approximate unknown regression functions". International Economic Review, 21: 149170. 
A Monte Carlo study on the fit of a cost function
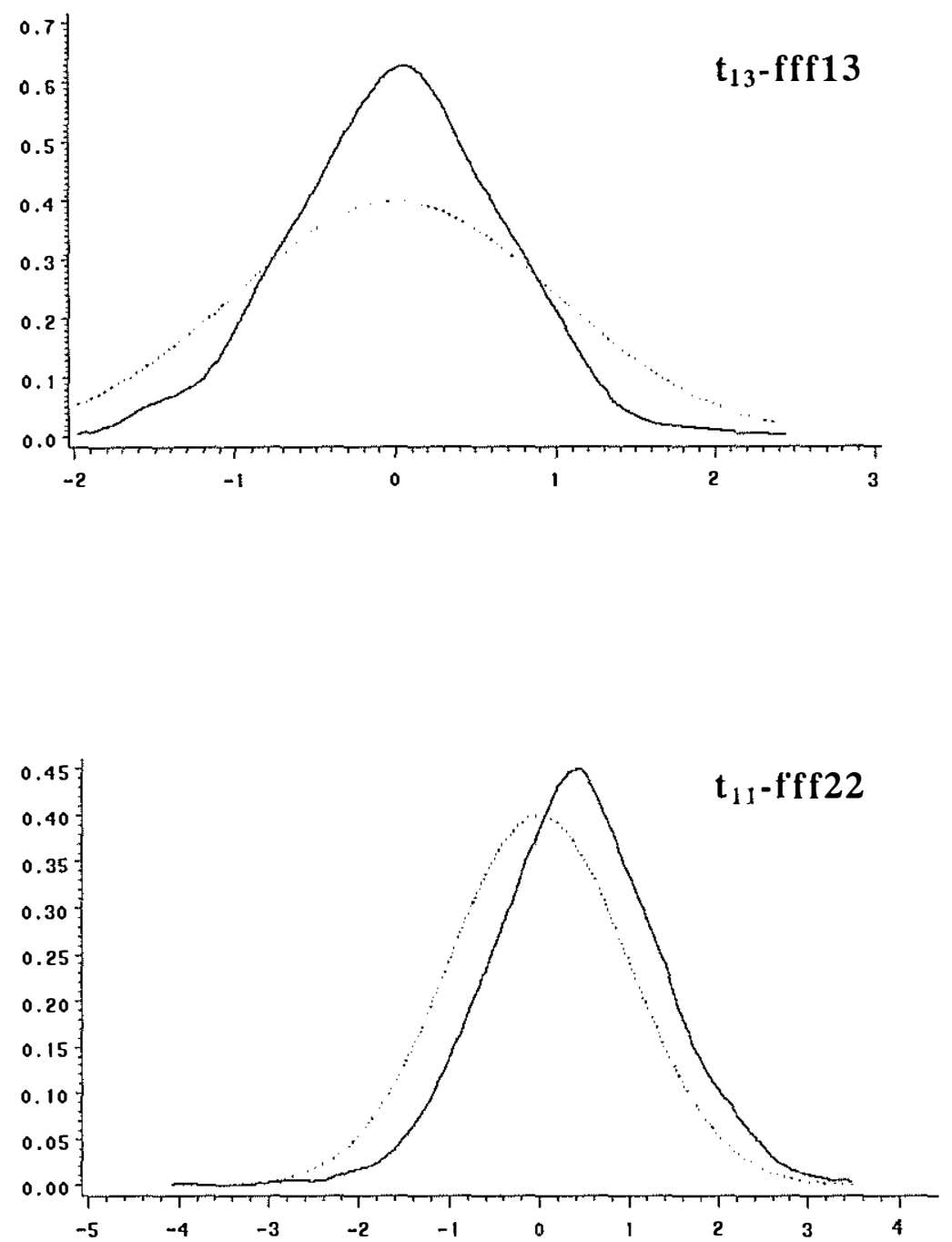

Figure 1: Nonparametric clensity estimates for the distribution of $t_{i j}$ for design 8. Normal kernel. The dashed curve is the standard normal 

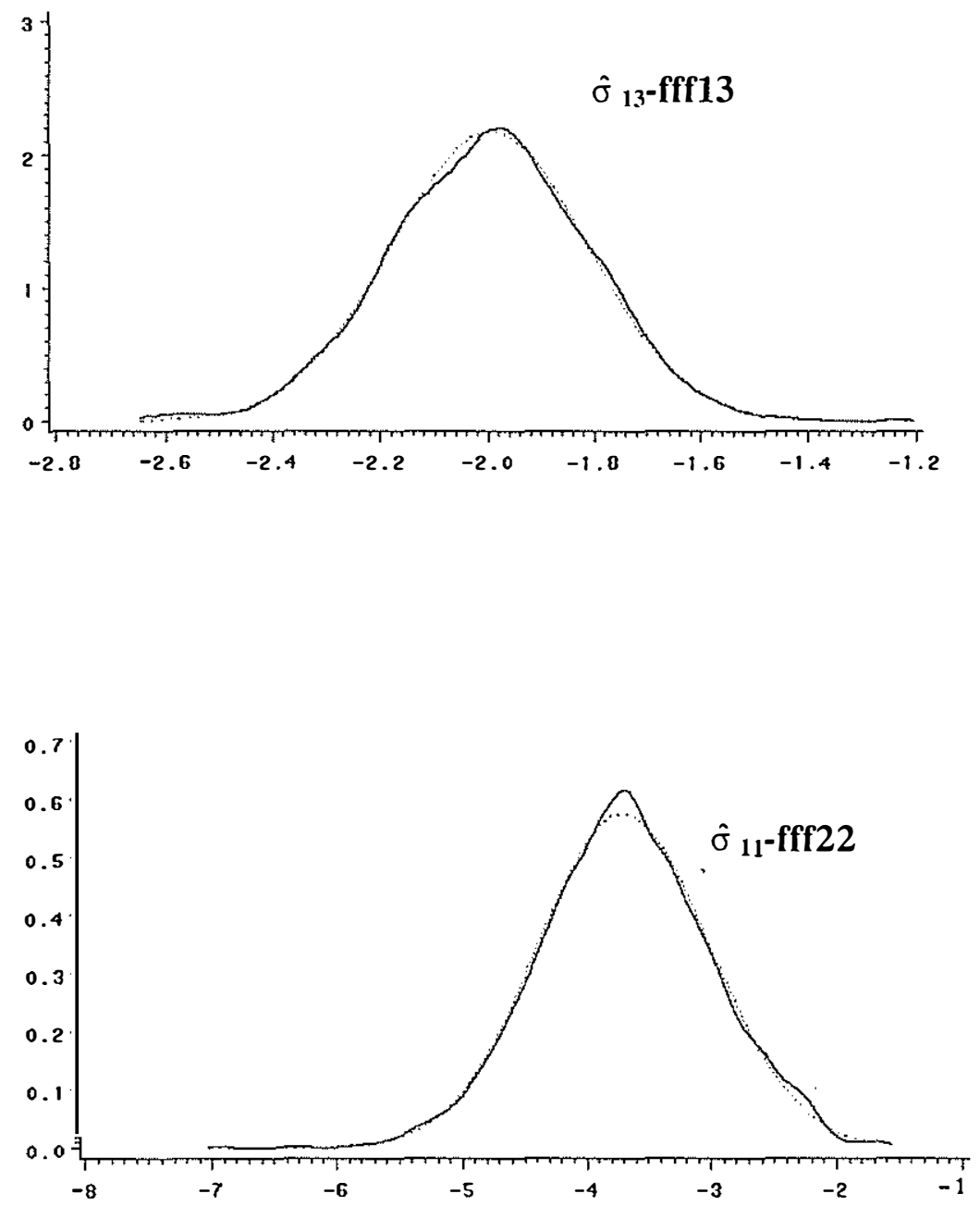

Figure 2: Nonparametric density estimates for the distribution of $\hat{\sigma}_{i j}$ for design 8. Normal kernel. The dashed curved is the normal density with mean and 
A Monte Carlo study on the fit of a cost function

Table 1

Population Technologies. For the Translog $\alpha_{0}=0.756928$, $\alpha_{1}=0.191614, \alpha_{2}=0.208386$, and $\alpha_{3}=0.6$

\begin{tabular}{|c|r|r|r|r|r|r|r|}
\hline \hline Design & Pattern & $\gamma_{11}$ & $\gamma_{12}$ & $\gamma_{13}$ & $\gamma_{22}$ & $\gamma_{23}$ & $\gamma_{33}$ \\
\hline \hline 1 & III & 0.0800 & -0.0800 & 0 & 0.0800 & 0 & 0 \\
2 & I & 0.1088 & 0.2294 & 0.1894 & 0.04003 & -0.0665 & 0.0275 \\
3 & I & 0.0667 & 0.3142 & 0.2266 & -0.0937 & -0.0342 & -0.0487 \\
4 & III & -0.0329 & 0 & 0.1495 & -0.0406 & 0.1660 & 0.0566 \\
5 & I & 0.2699 & 0.1302 & 0.0939 & 0.0405 & 0.0142 & 0.0211 \\
6 & III & 0.7896 & -0.2696 & -0.3167 & 0.8143 & -0.3216 & 1.1456 \\
7 & I & -1.0095 & 0.8086 & 0.7645 & 0.0401 & -0.6248 & 0.0358 \\
8 & II & -0.1088 & 0.4588 & -0.1263 & -0.3626 & 0.3992 & -0.0824 \\
9 & II & 0.1814 & 0 & 0 & 0.6043 & 0 & 0.1374 \\
\hline \hline
\end{tabular}

Table 2

Average Absolute and Relative Biases for the Fourier Flexible Form

\begin{tabular}{|c|c|c|r|r|}
\hline Design & $\begin{array}{c}\text { Absolute } \\
\text { Bias } \\
\text { FFF13 }\end{array}$ & $\begin{array}{c}\text { Absolute } \\
\text { Bias } \\
\text { FFF22 }\end{array}$ & $\begin{array}{c}\text { Relative } \\
\text { Bias } \\
\text { FFF13 }\end{array}$ & $\begin{array}{c}\text { Relative } \\
\text { Bias } \\
\text { FFF22 }\end{array}$ \\
\hline 1 & 0.00369950 & 0.0018189 & 1.0000 & 1.0000 \\
2 & 0.01527360 & 0.0232128 & 4.1286 & 12.7620 \\
3 & 0.00083742 & 0.0299361 & 2.2636 & 16.4584 \\
4 & 0.00730160 & 0.0820313 & 1.9737 & 15.0994 \\
5 & 0.02919300 & 0.0181192 & 7.8911 & 9.9616 \\
6 & 0.00802730 & 0.0098951 & 2.1698 & 5.4402 \\
7 & 0.00787500 & 0.0038115 & 2.1287 & 2.0955 \\
8 & 0.01153290 & 0.1398890 & 3.1174 & 76.9086 \\
9 & 0.00936490 & 0.0304001 & 2.5314 & 16.7135 \\
\hline \hline
\end{tabular}


Table 3

Response Surfore Analyssis - FFF13. Numbers in (.) are standard errors. Model is $\mid$ Bias $\mid=a_{0}+a_{1} \lambda+n_{2} \sigma+a_{3} \lambda^{2}+a_{4} \lambda \sigma+a_{5} \sigma^{2} \cdot R^{2}=0.1364$

\begin{tabular}{|l|c|c|c|c|c|}
\hline \multicolumn{1}{|c|}{ Source } & df & Sum of Scluares & Mlcau Square & F Ratio & Pr $>F$ \\
\hline Lack of Fit & 3 & 0.000985 & 0.000329 & 1.539 & 0.2175 \\
Pure Error & 45 & 0.009634 & 0.000214 & & \\
Total Ejror & 48 & 0.010622 & 0.000221 & & \\
\hline$a_{0}$ & $a_{1}$ & $a_{2}$ & $a_{3}$ & $a_{4}$ & $a_{5}$ \\
\hline-0.005830 & 0.029217 & 0.011425 & -0.007077 & -0.010333 & -0.002125 \\
$(0.010519)$ & $(0.015778)$ & $(0.015778)$ & $(0.007121)$ & $(0.006073)$ & $(0.007121)$ \\
\hline \hline
\end{tabular}

Table 4

Response Surfare Anulysis - FFF22. Numbers in (.) are standard errers. Nodel is $\mid$ Bias $\mid=a_{0}+a_{1} \lambda+a_{2} \sigma+a_{3} \lambda^{2}+a_{4} \lambda \sigma+a_{5} \sigma^{2} \cdot R^{2}=0.2354$

\begin{tabular}{|c|c|c|c|c|c|}
\hline \hline Source & $\mathrm{df}$ & Smin of Squares & Meau Scluare & F Ratio & $P r>F$ \\
\hline \hline Lack of Fit & 3 & 0.049123 & 0.016374 & 6.731 & 0.0008 \\
Pure Error & 45 & 0.109463 & 0.002433 & & \\
Total Error & 48 & 0.158586 & 0.003304 & & \\
\hline$a_{0}$ & $a_{1}$ & $a .2$ & $a_{3}$ & $a_{4}$ & $a_{5}$ \\
\hline 0.010201 & 0.027847 & -0.056332 & -0.005336 & 0.008950 & 0.037854 \\
$(0.040644)$ & $(0.060966)$ & $(0.06096(6)$ & $(0.027516)$ & $(0.023466)$ & $(0.027516)$ \\
\hline \hline
\end{tabular}

children father mother distribution munber response responsability correspondence construction

Table 5

Response Surface Analyssis - FFF13. Numbers in (.) are standard errors. Model is $\mathrm{m}_{1} \lambda^{2}=a_{0}+a_{1} \lambda+a_{2} \sigma+a_{3} \lambda^{2}+a_{4} \lambda \sigma+a_{5} a^{2} \cdot R^{2}=0.2163$

\begin{tabular}{|c|c|c|c|c|c|}
\hline Source & df & Sum of Scluares & Meau Scluare & F Ratio & $P r>F$ \\
\hline \hline Jack of Fit & 3 & 8.871097 & 2.957032 & 1.712 & 0.1781 \\
Pure Error & 15 & 77.738425 & 1.727521 & & \\
Total Error & 48 & 86.609522 & 1.804365 & & \\
\hline$a_{0}$ & $a_{1}$ & $a_{2}$ & $a_{3}$ & $a_{4}$ & $a_{5}$ \\
\hline 1.760963 & 1.739899 & 3.705639 & -0.836075 & -0.123121 & -1.392659 \\
$(0.949826)$ & $(1.424743)$ & $(1.424743)$ & $(0.643038)$ & $(0.548381)$ & $(0.643038)$ \\
\hline \hline
\end{tabular}


A Monte Carlo study on the fit of a cost function

Table 6

Response Siurfare

Model is hu $X^{2}=a_{0}+a_{1} \lambda+a_{2} \sigma+a_{3} \lambda^{2}+a_{4} \lambda \sigma+a_{5} \sigma^{2} \cdot R^{2}=0.3481$

\begin{tabular}{|l|c|c|c|c|c|}
\hline \hline Source & $\mathrm{d} f$ & Sum of Squares & Mlean Square & F Ratio & $P_{r}>F$ \\
\hline \hline Lack of Fit & 3 & $\overline{37.166524}$ & 12.388841 & 11.090 & 0.0000 \\
Pure Error & 45 & 50.271704 & 1.117149 & & \\
Total Error & 48 & 87.438228 & 1.821630 & & \\
\hline$a_{0}$ & $a_{1}$ & $\mathbf{a}$ & $\mathbf{a}_{3}$ & $\boldsymbol{a}_{4}$ & $a_{5}$ \\
\hline 1.915438 & 0.850512 & 4.156474 & -0.248192 & 0.129934 & -1.636403 \\
$(0.954359)$ & $(1.431543)$ & $(1.431543)$ & $(0.646107)$ & $(0.550999)$ & $(0.646107)$ \\
\hline
\end{tabular}

Table 7

Response Surfare Aunlyssis

Morlel is $\mathrm{H}^{\circ}=a_{0}+a_{1} \lambda+a_{20}+a_{3} \lambda^{2}+a_{1} \lambda \sigma+a_{5} \sigma^{2} \cdot R^{2}=0.2368$

\begin{tabular}{|l|c|c|c|c|c|}
\hline \multicolumn{1}{|c|}{ Source } & di & Sum ol Squares & Nleau Square & F Ratio & $P r>F$ \\
\hline \hline Lack of Fit & 3 & 0.006684 & 0.002228 & 3.993 & 0.0132 \\
Pure Error & 45 & 0.025108 & 0.000558 & & \\
Total Error & 48 & 0.031793 & 0.000662 & & \\
\hline$a_{0}$ & $a_{1}$ & $a_{2}$ & $a_{3}$ & $a_{4}$ & $a_{5}$ \\
\hline 1.003676 & -0.039486 & -0.082646 & 0.025174 & -0.000274 & 0.039628 \\
$(0.018198)$ & $(0.027297)$ & $(0.027297)$ & $(0.012320)$ & $(0.010507)$ & $(0.012320)$ \\
\hline
\end{tabular}

Table 8

Response Sulfare Analygsis - FPF22. Numbers in (.) arr stondard errors.

Nodel is $I F=a_{0}+a_{1} \lambda+a_{2} \sigma+a_{3} \lambda^{2}+a_{1} \lambda \sigma+a_{5} \sigma^{2} \cdot R^{2}=0.3089$

\begin{tabular}{|c|c|c|c|c|c|}
\hline Sontre & $\mathrm{d} \Gamma$ & Suni of Squates & Mean Square & F Ratio & $p$ \\
\hline $\begin{array}{l}\text { Lack of Fit } \\
\text { Plure Error } \\
\text { Total Error }\end{array}$ & $\begin{array}{r}3 \\
45 \\
48\end{array}$ & $\begin{array}{l}0.000740 \\
0.005969 \\
0.006709\end{array}$ & $\begin{array}{l}0.000247 \\
0.000133 \\
0.000140\end{array}$ & 1.859 & 0.1502 \\
\hline$a_{0}$ & 11 & $a 2$ & $n_{3}$ & $a_{4}$ & $a 5$ \\
\hline $\begin{array}{c}0.999465 \\
(0.008360)\end{array}$ & $\begin{array}{l}-0.025555 \\
(0.012539)\end{array}$ & $\begin{array}{l}-0.048292 \\
(0.012539)\end{array}$ & $\begin{array}{c}0.014612 \\
(0.005659)\end{array}$ & $\begin{array}{c}0.000101 \\
(0.004826)\end{array}$ & $\begin{array}{c}0.024548 \\
(0.005659)\end{array}$ \\
\hline
\end{tabular}

